\title{
MedienPädagogik
}

www. medienpaed.com

Zeitschrift für

Theorie und Praxis

der Medienbildung

ISSN 1424-3636

Themenheft Nr. 18: Neue Medien und individuelle Leistungsdarstellung -

Möglichkeiten und Grenzen von ePortfolios und eAssessments

\section{Einsatz von E-Portfolios bei der Qualifizierung pädagogischer Professionals in restriktiven Settings}

Dirk Jahn, Bernhard Trager und Karl Wilbers

\begin{abstract}
Der Beitrag zeigt den Einsatz von E-Portfolios bei der Qualifizierung pädagogischer Professionals in der beruflichen Bildung auf. Dabei werden als Referenz die Theorie der Selbstreflexion, des kritischen Denkens sowie des Konzeptwandels zugrunde gelegt. Es werden Möglichkeiten aufgezeigt, wie E-Portfolios auch in restriktiven Settings eingesetzt werden können. Bislang dominiert in der Literatur der Einsatz von E-Portfolios in der Schule bzw. in der Bildung von Lehrkräften. Restriktive Settings sind Settings, die für das formale Lernen nur kurze Zeiträume eröffnen, mit heterogenen Zielgruppen verbunden sind und die zum Teil ungünstige motivationale und kognitive Ausgangsvoraussetzungen mitbringen. Die dargestellten Ergebnisse fussen auf der Entwicklung, Erprobung und Erforschung eines solchen Settings, nämlich der Erprobung eines Qualifizierungskonzepts für pädagogische Professionals im Einzelhandel.
\end{abstract}

\section{Bisheriger Einsatz von E-Portfolios zur Qualifizierung pädagogischer Professionals}

An den verschiedenen Lernorten sind unterschiedliche Personen zur Unterstützung von Lernprozessen tätig, die hier als «pädagogische Professionals» bezeichnet werden. Zur Qualifizierung von pädagogischen Professionals werden in den letzten Jahren verstärkt E-Portfolios eingesetzt.

Insbesondere im angelsächsischen Raum sind elektronische Portfolios im Bereich der universitären Bildung von Lehrkräften verbreitet (Lorraine et al., 2007, S. 87101). Im Handbook of Research on ePortfolios (Jafari \& Kaufman, 2006) werden zahlreiche Fallstudien zum Einsatz digitaler Portfolios aufgeführt. Sie beziehen sich zu einem überwiegenden Teil auf den Kontext der universitären Lehrerbildung bzw. den Lernort Schule. Eingesetzt werden elektronische Portfolios beispielsweise an den kanadischen Universitäten von Quebec, Ottawa und Saint-Paul (Peters et al., 2006), Windsor (Wright \& Hartley, 2009), den US-amerikanischen Universitäten von Hawaii (Bartlett, 2006, S. 327-339), der University of Maryland (Huang, 2006, S. 503-519) und der Montana State-University-Northern (Richter, 2006, S. 551-557). Auch an der University of Otago, Neuseeland (Spronken-Smith \& Stein, 2009, S. 197-210), der Wake Forest University in North Carolina, USA (Cunningham, 2009, 
S. 121-140) sowie der University of Ulster, Nordirland und dem Trinity College, Dublin (McNair \& Marshall, 2006, S. 474-486) finden Portfolios Verwendung in der Ausbildung angehender Lehrerinnen und Lehrer. In einer seit 1998 andauernden länderübergreifenden Kooperation arbeiten das Institut für Lehrerbildung der Universität von Uppsala, Schweden die Stanford University sowie das Königliche Institut für Technologie (KTH), Stockholm um zusammen E-Portfolio-Arbeit kontinuierlich zu verbessern (O.A., 2009a) Hornung-Prähauser et al. (2007, S. 107-152) liefern einen - über die Lehrerbildung hinausgehenden Überblick - der Verwendung von E-Portfolios an Hochschulen in den USA, Grossbritannien sowie in Finnland und den Niederlanden.

Während also den USA und Grossbritannien eine Vorreiterrolle bei der Nutzung von elektronischen Portfolios zugeschrieben wird, etablieren sich diese in den europäischen Ländern wie den Niederlanden, Schweden, Finnland, aber auch in der Schweiz und Österreich zunehmend. Im deutschsprachigen Raum setzen u. a. die Pädagogische Hochschule St. Gallen (Christen \& Hofmann, 2008), das «Netzwerk für E-Portfolio in der Lehrerbildung Hessen», sowie die Universität Erlangen-Nürnberg, Lehrstuhl für Wirtschaftspädagogik und Personalentwicklung, auf den Einsatz von elektronischen Portfolios. Darüber hinaus setzen weitere Hochschulen EPortfolios ein, wie das Institut für Erziehungswissenschaften der Universität Kassel (Bosse et al., 2009), das Zentrum für Lehrerbildung und Schul- und Unterrichtsforschung (ZLF) der Universität Frankfurt/ Main, das Landesinstitut für Lehrerbildung und Schulentwicklung Hamburg (Bade et al., 2009). Insgesamt sind E-Portfolios in Deutschland bisher vergleichsweise wenig verbreitet (O.A., 2009b). Weiterhin ist festzustellen, dass digitale Portfolios vor allem in der Lehrerbildung eingesetzt werden. Ausserhalb von Schule und Hochschule (Preckel \& Aschwanden, 2010) werden E-Portfolios noch zögerlich eingesetzt. Dies gilt für die betrieblichen Aus- und Weiterbildung und für die Qualifizierung des betrieblichen Bildungspersonals.

Der - dominierende - Einsatz von E-Portfolios in der Lehrerbildung wird regelmässig durch begünstigende Faktoren erleichtert, die so in anderen Anwendungsbezügen nicht gegeben sind. Die gewählten Zeiträume sind in der Regel verhältnismässig lang, zum Beispiel ein oder mehrere Semester. Die Gruppe der Studierenden ist im Vergleich zu anderen Anwendungsfeldern vergleichsweise homogen, was etwa durch vergleichsweise einheitliche Zulassungsbedingungen, zum Beispiel das Abitur, abgesichert wird. Anders als bei Berufstätigen konkurrieren Lernaktivitäten nur in geringen Umfang mit Erwerbsaufgaben. Schliesslich können Prüfungsregelungen die Motivationslage der Studierenden beeinflussen. So können Studierende vergleichsweise einfach zu E-Portfolio-Leistungen extrinsisch motiviert werden. 


\section{Referenztheorien zur Gestaltung von E-Portfolios in restriktiven Settings}

Im Folgenden werden als Grundlage für die Gestaltung der E-Portfolios in restriktiven Settings die Referenztheorien des Konzeptwandels, der Selbstreflexion und des kritischen Denkens dargestellt.

\subsection{Theorie des Wandels von Konzepten als Basis für die Gestaltung von E-Portfolios}

Pädagogische Professionals sind bereits vor ihrer Ausbildung bzw. ihrer ersten Tätigkeit keine unbeschriebenen Blätter mehr, sondern verfügen über umfangreiche Erfahrung als Lernende, die diverse Bildungseinrichtungen durchlaufen haben. Blömeke (2004) spricht mit Bezug auf Lehrkräfte von «Vorannahmen, Einstellungen bzw. Erwartungen» (S. 64), verwendet aber mangels konsistenter Begrifflichkeiten den Begriff «beliefs». Dieser wird auch in der angelsächsischen Forschung unterschiedlich verwendet, wie aus dem Übersichtsbeitrag von Pajares (1992) hervorgeht. Seit Ende der achtziger Jahre werden Konzepte wie beliefs, aber auch «images of teaching» (Calderhead \& Robson, 1991) oder "teacher perspectives» (Goodman, 1988) verwendet. Anders als beispielsweise Medizinstudierende, die sich mit neuen Umgebungen wie Operationssälen oder Notaufnahmeräumen auseinandersetzen müssen, sind Lehramtsstudierende «insider in a strange land» ( $\mathrm{Pa}$ jares, 1992, S. 323). Beliefs haben eine grosse Bedeutung für die implizite Steuerung von Unterricht, sind erfahrungsbasiert und erweisen sich als stark änderungsresistent. Nach dem Review von Wideen, Mayer-Smith \& Moon (1998) wissen Studierende, wie Unterricht auszusehen hat. Die Studierenden haben beispielsweise ein einfaches Verständnis von Lehren als Informationstransfer. Die Frage nach der Änderung dieser beliefs bzw. dieser Präkonzepte führt zur Frage des Konzeptwandels (Vosniadou 2008). Der Prozess des Konzeptwandels kann nach Nissani \& Hoefler-Nissani (1992) in sechs Stufen beschrieben werden. Zunächst entsteht beim Lerner, hier dem pädagogischen Professional, ein initiales Unbehagen mit den vorhandenen Konzepten, woraufhin der Lerner versucht, die Inkonsistenzen zwischen dem Konzept und der diesbezüglichen Evidenz zu umgehen, beispielsweise durch Umdeutungsprozesse. Diesem folgen in der nächsten Phase des Konzeptwandels ein Zweifel am eigenen Konzept, ein Schwanken und schliesslich ein Wandel des Konzepts. Die Änderung von Präkonzepten verlangt eine Förderung bei der Explizierung von Vorannahmen, ein Sichtbarwerden von Unterschieden in Konzepten der Lerner, das Anregen von Metakognition an und das Explorieren des Status der Konzepte (Hewson, Beeth \& Thorley; 1998). 


\subsection{Die Selbstreflexionstheorie als Basis für die Gestaltung von E-Portfolios}

In der Ausbildung pädagogischer Professionals kommt der Förderung von Selbstreflexion eine besondere Bedeutung zu (Tusch \& Steinlechner, 1988; Dauber \& Zwiebel, 2006, Gudjons, Wagener-Gudjons \& Pieper, 2008). Ein einheitlicher Selbstreflexionsbegriff ist dabei nicht zu finden. Greif $(2008$, S. 20) sieht ergebnisorientierte Selbstreflexion als ein in jedem Menschen vorhandenes Potenzial zu bewusster Selbstveränderung: «Individuelle Selbstreflexion ist ein bewusster Prozess, bei dem eine Person ihre Vorstellungen oder Handlungen durchdenkt und expliziert, die sich auf ihr reales und ideales Selbstkonzept beziehen. Ergebnisorientiert ist die Selbstreflexion, wenn die Person dabei Folgerungen für künftige Handlungen oder Selbstreflexionen entwickelt» (Greif, 2008, S. 40). Damit hat die Selbstreflexionstheorie einen engen Bezug zur kontinuierlichen Kompetenzentwicklung bzw. lebenslangem Lernen. Dies lässt sich an folgendem zyklischen Phasenverlauf der Kompetenzentwicklung veranschaulichen (Schratz, 2006).

Ausgangspunkt ist ein Handeln im Zustand unbewusster Inkompetenz. Um dies bewusst zu machen, muss die Selbstaufmerksamkeit auf die Diskrepanzen im eigenen Handeln und Denken gelenkt werden (Greif, 2008, S. 35). Es folgt die zweite Phase, die der bewusst gewordenen Inkompetenz. Sie ist von Unsicherheit geprägt (Schratz, 2006, S. 30). Die alten, vertrauten Denk- und Handlungsmuster haben sich als unbrauchbar erwiesen, gleichzeitig müssen alternative Optionen jedoch erst noch erarbeitet werden. Über die Problemanalyse bzw. das bewusste Verstehen der Ursachen für die bisherige unbewusste Inkompetenz können nun neue Muster generiert werden. Im Idealfall ist die Person motiviert, wahrgenommene Kompetenzlücken zu schliessen, beispielsweise indem neue alternative Handlungsmuster erarbeitet und in das Handlungsrepertoire integriert werden (Tisdale, 1998; Trager, 2008, S. 80-90). Dies führt zur dritten Phase, die der bewussten Kompetenz. Neu erworbenes Wissen bzw. Kompetenzen werden allmählich immer mehr verinnerlicht werden. So wird zwar das Handlungsrepertoire erweitert, kurzfristig kann dies jedoch auch zu einer verlangsamten Ausführung von Tätigkeiten führen. Daher ist es für die Kompetenzentwicklung notwendig, dass den Lernenden Phasen eingeräumt werden, in denen sie möglichst frei von Zeit- und Ergebnisdruck mit neuen Mustern experimentieren können. Durch das zunehmende Verinnerlichen des neuen Musters ist es möglich die vierte Phase des Modells, den Zustand unbewusster Kompetenz zu erreichen.

Durch eine geeignete Gestaltung des Prozesses bieten E-Portfolios vielfältige Möglichkeiten, Selbstreflexion zu unterstützen. Gleichwohl wird das Potenzial oft nicht genutzt. So wird beispielsweise Reflexion als Kernelement der Arbeit mit Portfolios und E-Portfolios gesehen (Häcker, 2007; Danielson \& Abrutyn, 1997). Barrett (2005) bezeichnet Reflexion gar als «the heart and soul of the E-Portfolio». Insofern überrascht es, wenn Hilzensauer (2008) nach der Analyse verschiedener 
E-Portfolio-Software-Tools zu folgender Feststellung kommt: «Die Unterstützung reflexiven Lernens ist in den E-Portfolio-Software-Tools nur durch das Vorhandensein einer leeren Infrastruktur und ungenügend dokumentierter Funktionen vorhanden. Methodische Anleitungen dafür sind selten und nur von allgemeiner Natur. Darüber hinaus bezieht sich die Reflexion im weitesten Sinne nur auf den Lerngegenstand und selten auf die Lernhandlung. Das Lernvermögen bleibt unberührt» (S. 16).

\subsection{Die Theorie des kritischen Denkens als Basis für die Gestaltung von E-Portfolios}

Kritisches Denken beinhaltet nach Brookfield (1987, S. 7) als Kernelement die Analyse von Annahmen, die unkritisch übernommen werden und nicht auf Evidenz und überprüfte Schlussfolgerungen gestützt sind. Annahmen sind als für gegeben erachtete Vorstellungen und Überzeugungen über die Welt und über uns als Teil der Welt zu verstehen, sozusagen scheinbare Selbstverständlichkeiten, die als erwiesen erachtet und deshalb oftmals nicht hinterfragt werden. Annahmen können reine Glaubenssätze und Alltagstheorien sein, denen aber allgemeine Gültigkeit zugeschrieben wird. Aufgrund von Annahmen treffen Menschen Entscheidungen, legen sich Erklärungen zurecht, bilden Urteile oder nehmen selektiv wahr (Weil, 2004, S. 63). Dabei werden Annahmen in den meisten Fällen nicht ausgesprochen, sondern als selbstverständlich vorausgesetzt. Annahmen sind notwendig, um gedanklichen Schlussfolgerungen und den darauf fussenden Handlungen Sinn zu verleihen. Sie beeinflussen massgebend menschliches Denken und Handeln. Dadurch können sie sich jedoch potentiell irreführend und täuschend auf das Denken auswirken und zu falschen Interpretationen und schliesslich auch zu unangemessenen Handlungsweisen führen (Browne \& Keeley, 1986, S. 53). Pädagogisches Handeln und Denken wird beispielsweise durch Erfahrungen als Schüler und in der eigenen Praxis oder den im Studium oder Ausbildung vermittelten pädagogischen Paradigmen und pädagogischen Alltagsrezepten beeinflusst, die nicht immer hinreichend auf ihre Richtigkeit von dem pädagogischen Professional geprüft worden sind (Grant, 1988).

Kritisches Denken soll helfen, durch Identifikation, Analyse und Bewertung von Annahmen bewusster und sicherer urteilen, entscheiden, schlussfolgern und schliesslich handeln zu können (Ennis, 1996, S. xvii). Hierzu zählt besonders die Beleuchtung von gesellschaftlichen Regeln, Werten, Ereignissen oder auch Auswirkungen von Handlungen usw., die oftmals als «normal» - als selbstverständlich und als gegeben - betrachtet werden (Brookfield, 2003, S. 144). Da kritisches Denken zu emanzipierten Handlungs- und Denkweisen führen soll, ist es gekennzeichnet durch das in Betracht ziehen von fantasievollen Ideen und der damit verbundenen Erkundung von Alternativen und braucht neben Mut, Ausdauer und Fantasie vor allem die Bereitschaft, sich mit einer breiten Palette an verschiedenartigsten 
Sichtweisen und Meinungen auseinander zu setzen. Dabei gehen Reflexion und sozialer Austausch Hand in Hand. Zuletzt ist kritisches Denken durch einen reflexiven Skeptizismus geprägt, einer Neigung, Wahrheiten, die als universell angesehen oder proklamiert werden, zu hinterfragen. Diese Haltung soll aus der Analyse von Annahmen, der Bewusstmachung des Wirkens von Kontext auf unser Denken und dem Ersinnen von erfinderischen alternativen Ideen und deren Erkundung erwachsen und zur Emanzipation des Menschen von fremden Kräften führen (Brookfield, 1987, S. 15 ff.).

Kritisches Denken ist analytisch, d. h. dass das Denken an strengen Kriterien ausgerichtet werden muss wie z. B. Klarheit, Richtigkeit, Exaktheit, Relevanz oder Logik (Paul, 1993, S. 138). Kritisches Denken ist aber auch multiperspektivisch: Verschiedenste Annahmen zu einem Sachverhalt sollen aus dem weitestmöglich zur eigenen Perspektive entferntesten Winkel betrachtet werden, um die Komplexität und die Interdependenz der Realität zu verstehen. Kritisches Denken ist des Weiteren ideologiekritisch: Es reflektiert und analysiert bestehende soziale Normen und Verfahrensweisen im Hinblick auf verdeckte oder offene Herrschaftsformen, die Rolle von Macht und die Auswirkungen von instrumenteller Vernunft. In der Tradition der kritischen Pädagogik fragt es danach, wer durch etwas profitiert und wer dadurch in seiner freien Entfaltung als Mensch eingeengt oder gar unterdrückt wird (Burbules \& Berk, 1999, S. 46). Pädagogische Professionals in dieser Tradition würden ihre Praxis aus verschiedenen Blickwinkeln auf offene und verdeckte Formen von Macht und deren Wirkung hin untersuchen. Sie würden beispielsweise ihre eigene Einflussnahme auf Lerner als Autorität, die politischen und gesellschaftlichen Dimensionen, die in dem zu vermittelnden Wissen, welches der "offizielle» Lehrplan fordert, verborgen sind, aber auch die subdidaktische Wirkung von Methoden und Medien ideologiekritisch hinterfragen (McDonald, 1983, S. 303 f.; Brookfield, 2005, 117 ff.). Ziel dieser Analyse ist die Verwirklichung einer repressionsfreien und emanzipatorischen Praxis. Kritisches Denken ist also darüber hinaus in seiner Zielsetzung konstruktiv, d.h. dass es darauf ausgerichtet ist, alternative Handlungs- und Denkansätze zu schaffen, durch deren Integration die pädagogische Praxis verbessert werden kann.

\subsection{Gemeinsamkeiten und Unterschiede der Referenztheorien zur Gestaltung von E-Portfolios}

Die darstellten Referenztheorien des Konzeptwandels, der Selbstreflexion und des kritischen Denkens weisen deutliche Parallelen zueinander auf. Alle Ansätze tragen Potenziale zu Veränderung und Wandel in sich und sind erkenntnistheoretisch motiviert. Es geht darum, durch bewusste Wahrnehmung und ein tiefergehendes Verstehen eindimensionale Denk- und Handlungsmuster zu überwinden. Alle drei Referenztheorien setzen als Impuls und Initiierung der Prozesse eine Diskrepanzer- 
fahrung voraus, die ein Unbehagen, Verwirrung, Verwunderung, Provokation usw. als Startpunkt der Denkprozesse auslöst.

Kritisches Denken ist im Vergleich zur Selbstreflexion mehr auf die Identifizierung und Analyse von Annahmen bezogen. Es geht beim kritischen Denken mehr darum, sich wohlbegründete Urteile zu erschliessen, auf die kritisch antizipierte Entscheidungen folgen. Thematisch stehen in der Selbstreflexion die eigene Person und deren Handlungsrepertoire im Mittelpunkt der Denkrichtung. Kritisches Denken hingegen beschäftigt sich aber verstärkt mit dem Bilden von vorläufig «richtigen» Urteilen und dem Ableiten von wohlüberlegten Schlussfolgerungen (Moon, 2008, S. 145). Dabei kann natürlich das eigene Handeln, die eigene Person im Zentrum des Denkens stehen, jedoch sind auch alle weiteren Themenbereiche eingeschlossen, die erst einmal nicht unmittelbar mit der eigenen Person verbunden sein müssen. Dennoch zeigt sich, dass kritisches Denken und Selbstreflexion miteinander eng verwurzelt sind: Reflexion ist ein Prozess der Klärung, ein Umgehen mit unsicheren und unstrukturierten Erinnerungen, Eindrücken, Erlebnissen, Ideen und Gedanken. Ein Umgang mit solchen Strukturen regt gleichzeitig auch kritisches Denken an (Moon, 2008, S. 145). Kritisches Denken wiederum benötigt Selbstreflexion, um beispielsweise eigene Annahmen zu hinterfragen.

Kritisches Denken und der Wandel von Präkonzepten haben hohe Übereinstimmungen. Präkonzepte und Annahmen, die im kritischen Denken der Analyse unterworfen wurden, sind weitgehend deckungsgleich. Der Konzeptwandel versucht zu helfen, falsche Annahmen zu korrigieren und richtige zu generieren. Kritisches Denken zielt hingegen darauf ab, zu wohlbegründeten Urteilen und Entscheidungen zu gelangen. Selbstreflexion hat u. a. als Ziel, unbewusste Inkompetenzen bewusst zu machen und dadurch das eigene Handlungsrepertoire zu erweitern.

\section{Methodik}

Um die Gestaltung von E-Portfolios für pädagogische Professionals auf Basis der erwähnten Referenztheorien in einem restriktiven Setting zu untersuchen, wurde eine berufsbegleitende Weiterbildung für pädagogische Professionals aus der Aus- und Weiterbildung im Einzelhandel gewählt. Die berufliche Aus- und Weiterbildung im Einzelhandel ist heterogen und vollzieht sich an verschiedenen Lernorten, wie zum Beispiel den beruflichen Schulen, Unternehmen, überbetrieblichen Bildungsstätten, Weiterbildungsträgern u. a. Die Gruppe der pädagogischen Professionals ist ausgesprochen heterogen. Neben die grosse Gruppe der Lehrkräfte treten die pädagogischen Professionals im Betrieb. Es bereitet schon Schwierigkeiten, diese unter eine Bezeichnung zu fassen: «Ausbilder», "Berufspädagoge», «Bildungsberater» oder «Bildungscoach» sind Berufs- und Tätigkeitsbeschreibungen, die die differenzierte Situation des Berufsbildungspersonals in den Betrieben widerspiegeln (Diettrich \& Meyer, 2008). Die Qualifzierungsmassnahme wurde mit acht Teilnehmern im Alter von 25 bis 46 Jahren durchgeführt. Sie waren zum 
Zeitpunkt der Durchführung tätig an einer Berufsschule (1), den überbetrieblichen Bildungseinrichtungen des Handels (3), in Unternehmen (3) sowie einer Hochschule (1). Die Tätigkeitsbereiche der Teilnehmer erstreckten sich von den klassischen Aufgaben eines Berufsschullehrers, über die Tätigkeit eines E-Learning-Tutors, zwei Redakteurinnen für Online-Medien, eine Mitarbeiterin der E-Learning-Beratungsstelle einer Hochschule sowie drei Personalentwicklerinnen, die vor allem für die Auszubildenden ihres Unternehmens zuständig sind. Bis auf zwei Teilnehmer konnten alle auf eine universitäre Vorbildung zurückblicken. Die Teilnehmer verfügten bezüglich E-Learning allesamt ein für pädagogische Professionals in der beruflichen Bildung durchschnittliches bis sehr gutes Kenntnisniveau.

Die Durchführung erfolgte in einem Blended-Learning-Ansatz mit vier Präsenzterminen (einmal eintägig und dreimal zweitägig). Während der Online-Phasen, die zwischen zwei bis vier Wochen dauerten, erarbeiteten die pädagogischen Professionals die fachlichen Inhalte und führten parallel dazu in Weblogs Reflexionsaufträge aus. Grundlage für die Wissensvermittlung waren WBT-Module, die auf einer Lernplattform (ILIAS) zur Verfügung gestellt wurden. Als E-Portfolio-Tool wurde Mahara eingesetzt. Die Massnahme erstreckte sich über zwölf Wochen. Gleichzeitig wurde angestrebt, dass die Teilnehmer und Teilnehmerinnen sich gegenseitiges Feedback dazu geben sollten. Hierfür wurden Reflexionspartnerschaften in Gruppen auf der Lernplattform mit eigenen Foren eingerichtet. Die Zuteilung zu den Zweier- und Dreier-Gruppen erfolgte nach dem Zufallsprinzip. Die Teilnehmer konnten vor Beitritt in eine Gruppe nicht sehen wer bereits Mitglied war.

Die Datenerhebung erfolgte hauptsächlich mittels qualitativer Methoden, die durch standardisierte Erhebungen ergänzt wurden. Während und nach der Durchführung des Qualifizierungskonzepts wurden leitfadengestützte Interviews mit den Teilnehmerinnen und Teilnehmern des Qualifizierungskonzeptes durchgeführt. Hinzu kamen die teilnehmende Beobachtung während der Präsenztreffen und ebenso das Führen von Forschungstagebüchern.

\section{Gestaltung von E-Portfolios für die Weiterbildung von pädagogischen Professionals}

4.1 Übersicht über die Phasen der Gestaltung von E-Portfolios

Bei der (E-)Portfoliomethode handelt es sich um einen ausgesprochen komplexen Prozess, der an die beteiligten Lehrenden und Lernenden vergleichsweise hohe Anforderungen stellt. Hilzensauer \& Hornung-Prähauser (2006) beschreiben den EPortfolioprozess in fünf Phasen: 1. Klärung von Zielsetzung und Kontext für die digitale Portfolioarbeit, 2. Sammeln, Auswählen und Verknüpfen von Artefakten mit den Lernzielen, 3. Reflektieren und Steuern des Lernprozesses, 4. Präsentieren und Weitergeben der E-Portfolio-Artefakte, 5. Bewerten und Evaluieren von Lernprozessen. Für kurz- bis mittelfristig angelegte Qualifizierungsmassnahmen unter drei Monaten erscheint dieser Prozess in restriktiven Settings nach unserer Erfahrungen 
in dieser Form nicht durchführbar. Gemäss der Phasenfolge von Hilzensauer und Hornung-Prähauser (2006) bedarf es beispielsweise nach der Klärung des Zielkontextes zunächst des Sammelns von Artefakten bevor in die Reflexion eingestiegen werden kann. Daher wurde der Versuch unternommen, den E-Portfolioprozess für die Anforderungen eines Kurzzeitsettings bei der Qualifizierung pädagogischer Professionals zu modifizieren, und zwar so, dass die Vorteile der E-Portfoliomethode für die Förderung von Selbstreflexion, kritischem Denken und Konzeptwandel genutzt werden können. Vor allem das reflexive Schreiben, die multimedialen Darstellungsmöglichkeiten (Videos etc.), die Feedbackmöglichkeiten und weitere netzbasierte Werkzeuge wie Foren (Yang, 2007) können zur Förderung des kritischen Denkens (Yang, 2007), der Selbstreflexion und dem Konzeptwandel unterstützend beitragen. Moderne E-Portfolios wie Mahara beinhalten zum Grossteil auch weitere synchrone und asynchrone Kommunikationswerkzeuge.

Neben der Tatsache, dass die Kursdauer für die Umsetzung des klassischen E-Portfolioprozesses (Hilzensauer \& Hornung-Prähauser, 2006) zu kurz erschien, bestand eine weitere Restriktion darin, dass die meisten Teilnehmer reflexionsungewohnt waren. Angesichts dieser restriktiven Bedingungen wurde eine Sonderform des E-Portfolio-Prozesses entwickelt, die als «dissonanzgetrieben» bezeichnet werden kann. Sie ist speziell auf kurzfristige Weiterbildungsszenarien ausgerichtet, in denen Selbstreflexion, kritisches Denken und Konzeptwandel eine bedeutende Rolle spielen. Charakteristisch ist dabei eine sehr kurze Zeitspanne, die für die Heranführung an komplexe Konzepte und die Implementierung eines Feedback-Systems durch Reflexionspartnerschaften zur Verfügung steht. Dabei wird Dissonanz durch reflexionsanregende Arbeitsaufträge erzeugt, die anschliessend in Form von BlogPostings reflexiv aufgearbeitet wird. Hinzu kommen Aufgabenstellungen während der Präsenzveranstaltungen innerhalb des Blended-Learning-Konzeptes.

\subsection{Vermittlung des Nutzens von Selbstreflexion, kritischem Denken und Konzeptwandel}

Aus der Sicht der Selbstreflexionstheorie und der Arbeiten zu kritischem Denken sollten vor der eigentlichen E-Portfolioarbeit wichtige Klärungen erfolgen. Beispielsweise ist die berufliche Kompetenzentwicklung zu thematisieren und dabei der Zusammenhang zwischen Selbstreflexionsprozessen und der Erweiterung des Handlungsrepertoires zu erläutern. Vor der Bearbeitung des ersten Reflexionsauftrags im E-Portfolio wurden die Teilnehmer anhand von Beispielen aus dem pädagogischen Kontext (Rosenthal-Effekt, Präkonzepte bei Pädagogen etc.) und theoretischen Hintergründen zu den Referenztheorien motiviert. Durch das zunehmende Verständnis der Konzepte sollte die Akzeptanz und die Bereitschaft der Teilnehmer für Konzeptwandel, Selbstreflexion und kritisches Denken wachsen. Für die Heranführung an kritisches Denken lässt sich darüber hinaus feststellen, dass neben der konkreten Darstellung des Nutzens (Petri, 2003), der Erarbeitung 
eines Verständnisses von kritischem Denken in der jeweiligen Domäne (Meyers, 1986), der Vermittlung des theoretischen Fundaments aber auch bestimmte Diskussionsregeln benötigt werden, um das Diskutieren und Reflektieren kritisch und konstruktiv werden zu lassen (Brookfield \& Preskill, 2005).

Zum einen ist es wichtig, dass die pädagogischen Professionals analytisch über ihre Erfahrungen und Ansichten, beispielsweise zu kritischen Ereignissen aus Ihrer Praxis reflektieren. Das bedeutet, dass das Denken an den damit verbundenen Kriterien wie Relevanz, Evidenzbasiertheit, Klarheit, Exaktheit usw. ausgerichtet wird, um Diskussionen und Reflexionen mehr Substanz für Diskussionen zu verleihen. Zum anderen gilt es, Inhalte aus verschiedenen Perspektiven zu analysieren und ideologiekritische Fragen zu stellen, beispielsweise zu Wirkweisen eines heimlichen Lehrplanes, der Rolle von Macht und Hegemonie in Lernszenarien, Instrumentelle Vernunft bei dem Einsatz von E-Learning-Tools usw. Trotz der Vermittlung des Nutzens und der Kriterien für kritisches Denken sowie einem Input zum Geben von Feedback gelang es aber nicht, eine kritische Kommunikationskultur bei allen Teilnehmern zu etablieren. Eine der Teilnehmerinnen sahen sich beispielsweise nicht autorisiert genug, anderen ein kritisches Feedback zu geben. Nur Experten, die sich in der jeweiligen Materie auskennen, so ihre Auffassung, seien dazu berechtigt, kritisches Feedback zu geben.

\subsection{E-Portfolioarbeit einleiten}

Wesentlich für einen gelungenen Start waren die Schaffung einer förderlichen Lernatmosphäre sowie die Heranführung an die technischen Funktionen der EPortfolio-Software Mahara. Die Lernprozessqualität muss dabei von gegenseitigem Vertrauen und Akzeptanz sowie von Offenheit geprägt sein. In einem Kontext, in welchem die Lernatmosphäre von Misstrauen geprägt ist, erscheint es ausgeschlossen, dass Menschen bereit dazu sind, ihre Reflexionen über vorhandene Inkompetenzen bzw. individuelle Schwächen und damit verbundene Äusserungen von Emotionen u.ä. zu offenbaren. Selbst wenn das persönliche Verhältnis zwischen den beteiligten Personen als adäquat betrachtet wird, kann im Hintergrund die Angst schweben, ein Dritter, z. B. der Arbeitgeber, könnte unerlaubt und unerwartet Einblick nehmen. Im Kern geht es bei der Heranführung darum, vorhandenem Misstrauen mit Offenheit und Transparenz zu begegnen. Eine der grössten Herausforderungen im Rahmen des Settings war es, innerhalb kurzer Zeit die beschriebenen Variablen der Prozessqualität herzustellen. Das gegenseitige erste Kennenlernen der Teilnehmer, bereits vor dem ersten Präsenztreffen, erfolgte durch ein Online-Kennenlernspiel. Dieses Vorgehen wurde von den Teilnehmern positiv bewertet.

Des Weiteren sollten die pädagogischen Professionals bereits vor dem ersten Präsenztreffen mit den Funktionen der E-Portfolio-Software Mahara in einem sogenannten «Mahara-Führerschein» vertraut gemacht werden. Dazu wurde eine Anlei- 
tung erstellt, in der die grundlegenden Funktionen, wie Erstellung eines eigenen Profils, Hochladen von Dateien, Erstellen eines Blogs sowie von Ansichten und die Vergabe von Zugriffsrechten auf die Ansichten erklärt wurden. Die Aufgaben sollten dann durch die Teilnehmer durchgeführt werden. Die Auswertung dieser Vorgehensweise war nur teilweise zufriedenstellend. Die Anleitung (Mahara-Führerschein) wurde als zu grossschrittig kritisiert. Dennoch ist es sinnvoll, die Grundfunktionen nochmal in einer Präsenzveranstaltung zu thematisieren, z. B., um nach wie vor - unsichere Teilnehmer zu identifizieren und gezielt unterstützen zu können.

In der ersten Präsenzveranstaltung stellte sich ferner heraus, dass es sehr wichtig ist, den Teilnehmern Raum für die Äusserung vorhandener Bedenken einzuräumen. Am wirksamsten ist es hier, gut zuzuhören und Bedenken ernst zu nehmen. Auch wenn sich dies banal anhören mag, sollte es gerade aus medienpädagogischer Sicht erwähnt werden, denn: Die Teilnehmer fanden diese Grundeinstellung äusserst positiv und äusserten zugleich, dass sie sich in der Vergangenheit in anderen E-Learning-Kontexten von «übereifrigen IT-Enthusiasten» oft nicht ernst genommen fühlten, was sich auf ihre Motivation negativ ausgewirkt habe.

Eine weitere Massnahme, welche die Akzeptanz von elektronischen Portfolios fördern sollte war, dass die Kursleiter gewissermassen «in Vorleistung» gingen indem sie jeweils ein eigenes E-Portfolio führten. In der Einstiegsmaske der E-PortfolioPlattform stellten die Dozenten zudem in einem kurzen Video-Clip sich selbst sowie das E-Portfolio-Konzept vor. Das diente einerseits dem Kennenlernen - es wurde u. a. ein umfangreiches persönliches Profil eingestellt - und sollte andererseits zeigen, dass die Dozenten selbst Vertrauen in das Tool haben. Darüber hinaus hatte es den Nebeneffekt, dass die Teilnehmer eine bessere Vorstellung eines «gewachsenen» E-Portfolios bekommen konnten. Die Reaktionen darauf waren gemischt. Einerseits fanden es manche Teilnehmer förderlich, gleichzeitig fühlten sich andere wiederum «erschlagen» von derart umfangreichen E-Portfolios.

Zusammenfassend ist zu sagen: Eine intensive Heranführung an die E-PortfolioTechnik sowie den E-Portfolioprozess erfordert viel Aufwand und insbesondere viel Einfühlungsvermögen. Gleichzeitig entscheiden diese Bemühungen massgeblich mit darüber, ob der E-Portfolio-Prozess zur Förderung von kritischem Denken, Selbstreflexion und Konzeptwandel erfolgreich implementiert werden kann.

\subsection{Initiierung von Dissonanz}

Zur Förderung des kritischen Denkens, der Selbstreflexion und des Konzeptwandels benötigt es eingangs einen Impuls, der sowohl aus der eigenen Praxiserfahrung als pädagogischer Professionals entspringen, aber auch extern durch bestimmte Methoden erzeugt werden kann. Es gilt dabei, ein initiales Unbehagen mit vorhandenen Konzepten und Denkweisen anzuregen bzw. Diskrepanzen im eigenen Handlungsrepertoire bewusst zu machen. Die pädagogischen Professionals benö- 
tigen eine Diskrepanzerfahrung - sowohl positiver als negativer Art denkbar - bei der beispielsweise durch zwei oder mehrere Gedanken eine logische Inkonsistenz entsteht und dabei verinnerlichte Normen verletzt werden. Normen und Handlungen bzw. Gedanken sind nun nicht mehr miteinander vereinbar und widersprechen sich (siehe hierzu Beckmann, 1984, S. 10 ff). Im beschriebenen Projekt gab es mehrere Versuche, durch verschiedenartige Impulse Lernprozesse zu initiieren.

Zum einen wurde ein Lernmodul entwickelt, welches verschiedene Vorstellung von gutem Training und Unterricht aus Sichtweise verschiedener Lerntheorien und Modelle darlegt, dargestellt durch zugespitzte und provokante Persönlichkeiten, die per Videobotschaft zu einem bestimmten wirtschaftlichen Thema ihre radikale Sichtweise auf Bildung, Training und Unterricht, Lernen, die Lernenden usw. offenbaren. Bei der Bearbeitung des Modules mussten die pädagogischen Professionals die expliziten und impliziten Annahmen der jeweiligen Charaktere analysieren und anschliessend im E-Portfolio dazu eine leitfadengestützte Reflexion durchlaufen, die durch Hinterfragen der eigenen Annahmen bezüglich verinnerlichter Lerntheorien und Schülerbilder den Umgang mit institutionellen Bedingungen anregte und zum Argumentieren einlud.

Besonders die ideologiekritische Perspektive des kritischen Denkens findet allzu oft wenig Beachtung. Ein Beispiel ist die «Kevin-Studie», die zeigt, wie Schüler nur durch ihren Vorannahmen bei Lehrern verschiedene Verhaltensweisen hervorrufen, die sich in Bevorzugung bis Benachteiligung durch unterlassene Förderung gegenüber der Schüler niederschlagen (Trenkamp, 2009). Ziel war es deshalb, die pädagogischen Professionals für den Umgang mit Macht und Hegemonie für eine ideologiekritische und emanzipierende Praxis zu sensibilisieren. Hierfür wurden die Teilnehmer mit einem provokativen und in seiner Argumentation einseitigen Text zum «heimlichen Lehrplan» bzw. "Hidden Agenda» konfrontiert. Der heimliche Lehrplan wird hier als eine Art subdidaktische Kraft dargestellt, die unterhalb des eigentlichen Lehrplans wirksam ist und zu Verhaltenskonformität und leistungsbezogener Konkurrenz bei den Schülern führt (Weinz, 2003, S. 2). Der Text zielte darauf ab, eine dissonanzgetriebene Reflexion mit Leitfragen anzustossen, die via E-Portfolio durchgeführt wurde. Gerade diese eher fast schon selbst ideologische Schrift entfaltete im Hinblick auf seine Wirkung starke Betroffenheit, selbst bei erfahrenen pädagogischen Professionals. Eine der Teilnehmerinnen aus dem Personalbereich teilte beispielsweise mit, dass der Text sie sehr aufgewühlt habe und sie nun fast hinter allem pädagogischen Handeln einen heimlichen Lehrplan erkenne.

Eine weitere Aufgabe bestand darin, die Praktiker zu einem vorgegeben «kritischen» Fragenkatalog über ihre Erfahrungen in der Praxis im Portfolio reflektieren und schliesslich diskutieren zu lassen, wie in einem Audit. Sie setzten sich inhaltlich mit in dieser Zeit gemachten kritischen Erfahrungen in ihrer Praxis auseinander, die sie natürlich nur selbst kennen und daher selber wählen können, z. B. Dinge, die in 
ihrer Praxis schlecht gelaufen sind und sie frustriert, gedemütigt, verwundert usw. haben. In den offenen Fragen der Aufgabe ging es nun unter anderem darum, welche Entscheidungen die pädagogischen Professionals in der Praxis getroffen, wie sie in bestimmten Situationen gehandelt haben und welche Annahmen hinter ihrem Denken und Handeln stehen. Sie wurden aufgefordert, darüber kritisch nachzudenken, wie ihre Annahmen herausgefordert und in Frage gestellt wurden, ob sie aufgrund der Erfahrungen unter Umständen Annahmen revidieren mussten und welche neuen Wege folglich in der Praxis beschritten werden können oder wurden. Dabei wurden sie auch zu einer Perspektivenerweiterung und Überprüfung ihrer Annahmen sowie zum Erkunden alternativer Interpretationen des Ereignisses eingeladen, um so in Zukunft differenzierter mit derartigen Situationen umgehen zu können. Für pädagogische Professionals erachtet Brookfield hierbei vier Perspektiven der Reflexion für die eigene Praxis als relevant: 1. die eigene Autobiographie als pädagogischer Professional und Lerner und deren Einfluss auf die eigene Praxis (siehe dazu auch «beliefs» bei der Theorie des Konzeptwandels), 2. die Perspektive der Lerner («Walk in their shoes»), 3. Erfahrungen und Ansichten der Kollegen und 4. Theorien zum Lehren und Lernen (Brookfield, 1995, S. 29 ff.). An jeweils zwei Präsenzterminen wurde mit den Teilnehmern in Form von offenen Aufgaben gearbeitet, die den Charakter von Selbsterfahrungsübungen hatten. Auch damit sollte die Selbstreflexion angeregt werden. Die Teilnehmer wurden dabei u. a. gebeten, ihre eigene Lernbiografie mit einem Seil darzustellen und mit Metaplan-Karten zu ergänzen, wobei grüne Karten für Ressourcen und rote für Stolpersteine der eigenen Lernbiografie standen. Die pädagogischen Professionals erlebten diese Übung als ausserordentlich positiv. Nach ihren Worten wurde innen dabei der konkrete Nutzen von Selbstreflexion noch deutlicher, als durch Selbstreflexionsaufträge in Form von Fragestellungen. Zudem regte die Vorstellung und Besprechung der einzelnen Lernbiografien das nochmalige Nachdenken über die eigenen Stärken und Schwächen im eigenen Lernen an. Diese Reflexionen wurden später in das E-Portfolio inklusive Artefakte wie Bilder der Karten überführt und noch einmal vertiefend aufgegriffen.

Schreiben generell und die schriftliche Auseinandersetzung mit verschiedenen Weltsichten und den dahinter liegenden Annahmen bieten hervorragende Anlässe zur Förderung von kritischem Denken (Meyers, 1986, S. 69 ff.). Neben der individuellen Reflexion sollten sich die teilnehmenden pädagogischen Professionals auch in Zweier-Gruppen gegenseitiges Feedback geben. Die Perspektivenerweiterung bei der Selbstreflexion sowie im kritischen Denken benötigt soziale Interaktion (Offermanns, 2004; Greif, 2008). Das E-Portfolio eignet sich dabei auch als Kommunikationsmedium, da neben der Feedbackfunktion vor allem die Erstellung von Ansichten und die Regelung von Zugriffsrechten den Teilnehmern die benötigte Privatsphäre ermöglichte. So konnten eher intime Gedanken nur vertrauten Personen zugänglich gemacht werden. Der benötigte Austausch fand aber leider 
aus mehreren Gründen wie Arbeitsbelastung im Unternehmen etc. keineswegs so intensiv wie gewünscht statt.

\subsection{Unterstützung der Prozesse der Selbstreflexion, des kritischen Denkens und des Konzeptwandels}

Nach Offermanns (2004) ist es wichtig, dass Selbstreflexionsprozesse Unterstützung erfahren. Beispielsweise, um eigene Annahmen widerzuspiegeln, weitere Denkanstösse zu geben, Hoffnung und Zuversicht trotz Dissonanz zu vermitteln usw. Zur Aktivierung von Selbstreflexionsprozessen steht Lehrenden ein grosses Methodenrepertoire zur Verfügung. Entscheidend ist dabei, dass den Lernenden der Zugang zu den eigenen impliziten Annahmen, Normen und Zielen (Selbstzugang) erleichtert wird, beispielsweise durch Fragetechniken (z. B. zirkuläre Fragen) oder auch bildhafte Methoden (z. B. eines Selbstbildes in einer beruflichen Situation) (Greif, 2008). Auch die Modellierung von kritischem Denken, Selbstreflexion und Konzeptwandel durch «Modelle» wie Kursleiter, Teilnehmer usw. ist eine wichtige Unterstützungsstrategie zur Motivation und Demonstration von Denkprozessen. Modellieren bedeutet, eigene kritische Denkprozesse als pädagogischer Professional zu verbalisieren, um Schlussfolgerungen, Annahmen, verschiedene Perspektiven, Lösungsansätze und alternative Interpretationen zu gegebenen Fakten beobachtbar zu machen. Gerade E-Portfolios bieten für die Modellierung von Selbstreflexion, kritischem Denken und Konzeptwandel einige wesentliche Vorteile.

So wurden im Projekt Reflexionen der Kursleiter als Modelle genutzt. Einige Teilnehmer - vor allem die ohne universitären Hintergrund - fühlten sich jedoch durch allzu viel «pädagogisches Fachchinesisch» überfordert. Das Prinzip der Modellierung sollte also zielgruppengerecht eingesetzt werden. Beispielsweise sollten den verschiedenen Teilnehmern durch die Ansichtenfunktion im Portfolio auf die Zielgruppe abgestimmte, individuelle Beispielarbeiten zugänglich gemacht werden. Da das Handeln im Berufsalltag häufig von Zeit- und Ergebnisdruck geprägt ist, Prozesse der Selbstreflexion und des kritischen Denkens aber Zeit und Ruhe brauchen, besteht eine weitere wichtige Aufgabe pädagogischer Professionals darin, Lernende im E-Portfolio-Prozess kontinuierlich zu kritischen, selbstreflexiven Denkprozessen anzuhalten. Dies wurde durch laufende Reflexionsaufgaben, Feedback in den Lernpartnerschaften und durch aktivierende Fragen der Kursleiter unterstützt. Dabei half enger Kontakt zu den Teilnehmern, Erinnerungsmails, feste Vereinbarungen u. a. darüber, bis wann bestimmte Reflexionen getätigt werden müssen usw. Artefakte aus den Reflexionsübungen der Präsenzveranstaltungen wie etwa Bilder der einzelnen Lernbiografien wurden fotografiert und zur weiteren Reflexion und Diskussion für das E-Portfolio zur Verfügung gestellt, Ideen und Erkenntnisse dadurch im Portfolio weiterentwickelt. 


\subsection{Abschluss und Integration}

Um den Prozess am Ende einer kurz- bis mittelfristig angelegten Qualifizierungsmassnahme zu einem konstruktiven Abschluss zu bringen, mussten die zuvor ausgelösten Dissonanzen durch Integration von neu erworbenen Handlungs- und Denkmustern aufgelöst werden. Am Ende des Qualifizierungskonzeptes sollten die im E-Portfolio erstellten Reflexionen in Bezug auf Selbstreflexion, kritisches Denken und Konzeptwandel in das zukünftige pädagogische Handeln integriert werden. Somit soll sozusagen der Kreis geschlossen werden. Dies ist insbesondere deshalb wichtig, weil zwar einerseits die erzeugte Dissonanz wichtig für die Auslösung von Reflexionsprozessen ist, andererseits Lernende aber nicht in diesem Zustand verbleiben sollten. Blieben die pädagogischen Professionals in diesem Dissonanzzustand verhaftet, wäre mit einer gewissen Wahrscheinlichkeit die Handlungsfähigkeit gefährdet (Greif, 2008). Eine wichtige Rolle spielt dabei die Präsentation der EPortfolios (Häcker, 2007, S. 148). Am Ende des E-Portfolioprozesses soll ein Ergebnis sichtbar sein, das insbesondere den entstandenen Lernerfolg greifbar macht. Wichtig ist darüber hinaus die Frage, wie es mit dem in der Weiterbildungsmassnahme entstandenen E-Portfolio weitergeht. Im Projekt Flexible-Learning war zu beobachten, dass manche Teilnehmer gegen Ende des Kurses unaufgefordert eigenständige Blog-Beiträge zu Selbstreflexionen, kritischem Denken und Konzeptwandel erstellt haben. Dies legt die Schlussfolgerung nahe, dass es bei diesen Personen gelang, den Nutzen dieses E-Portfolio-Settings zu vermitteln. Andere Teilnehmer wiederum hatten Mühe, die vorgesehenen Beiträge anzufertigen. Insgesamt zeigten die abschliessenden Interviews, dass die Versuche, Selbstreflexion, kritisches Denken und Konzeptwandel in einem Kurzzeitsetting mit Hilfe von E-Portfolios zu unterstützen, vielversprechend und teilweise überaus erfolgreich waren. In künftigen Durchführungen muss die Menge an Reflexionsaufträgen im Verhältnis zur Kursdauer deutlich reduziert werden. Darüber hinaus gilt es Wege zu finden, wie Reflexionspartnerschaften besser initiiert werden können. Eine zentrale Erkenntnis der Durchführung ist die grosse Bedeutung, die die Heranführung an die E-Portfolioarbeit hat.

\section{$5 \quad$ Fazit}

Zusammenfassend kann festgestellt werden, dass E-Portfolios auch in restriktiven Settings ausserhalb von Schule und Hochschule erfolgversprechend eingesetzt werden können. Allerdings gilt es hierfür, einigen Punkten besondere Aufmerksamkeit zu schenken. Entscheidend ist in erster Linie die Einstiegsphase. In restriktiven Settings, wie dem beschriebenen, verbleibt nur sehr wenig Zeit und Gelegenheit für die Vermittlung der technischen Funktionen, die Heranführung an die komplexe E-Portfolio-Methode sowie - vor allem - die Schaffung einer vertrauensvollen und damit reflexionsförderlichen Lernatmosphäre. Eine weitere Herausforderung besteht darin, die für Konzeptwandel, Selbstreflexion und kritisches Denken er- 
forderliche Dissonanz herzustellen und konstruktiv zu lenken. Der beschriebene dissonanzgetriebene E-Portfolio-Prozess ist als Sonderform des von Hilzensauer \& Hornung-Prähauser (2006) beschriebenen E-Portfolio-Prozesses zu verstehen, bei dem der Schwerpunkt auf der Förderung von Konzeptwandel, Selbstreflexion und kritischem Denken liegt.

\section{Literatur}

Bade, Peter; Hitter, Kirsten; Köhn, Wiebke; Kradel, Hans-Dieter; Nitschkowski, Angela; Seyd, Christopher. «Das Portfolio im Referendariat - Hamburger Modell. Handreichung für Referendarinnen und Referendare.» Landesinstitut für Schulentwicklung und Lehrerbildung Hamburg (2009): http://www.li-hamburg.de/fix/ files/doc/LIA\%202009_10\%20Portfolio\%20Web.pdf (23.12.09).

Bartlett, Andrea. «lt Was Hard But It Was Worth It: ePortfolios in Teacher Education». Handbook of Research on ePortfolios. Hrsg. v. Ali Jafari \& Catherine Kaufman. London: Idea Group, 2006. 327-339.

Barrett, Helen. «E-Portfolios: Digital Stories of Lifelong and Lifewide Learning.» electronicportfolios.com (2005): http://electronicportfolios.org/portfolios/Eifel2005.pdf (10.7.09).

Beckmann, Jürgen. Kognitive Dissonanz: Eine handlungstheoretische Perspektive. Berlin: Springer-Verlag, 1984.

Blömeke, Sigrid. «Empirische Befunde zur Wirksamkeit der Lehrerbildung.» Handbuch Lehrerbildung. Hrsg. v. Sigrid. Blömeke, Peter Reinhold, Gerhard Tulodziecki \& Johannes Wild. Bad Heilbrunn/Obb: Klinkhardt, 2004. 59-91.

Bosse, Dorit; Technau Bastian; Junghans, Markus; Frommann, Uwe. «E-Portfolios in der Lehrerbildung.» Universität Kassel. Institut für Erziehungswissenschaft, 2009: http://cms.uni-kassel.de/unicms/index.php?id=27989 (23.12.09).

Brookfield, Stephen. The Power of Critical Theory: Liberating Adult Learning and Teaching. San Francisko: Jossey-Bass, 2005.

Brookfield, Stephen; Preskill, Stephen. Discussion as a Way of Weaching: Tools and Techniques for Democratic Classrooms. San Francisco: Jossey Bass, 2005.

Brookfield, Stephen. "Critical Thinking in Adulthood.»Critical Thinking and Reasoning. Current research, Theory, and Practice. Hrsg. v. Daniel. J. Fasko. New Jersey: Hampton Press, 2003. 143-163.

Brookfield, Stephen. Becoming a Critically Reflective Teacher. San Francisco: Jossey-Bass, 1995.

Brookfield, Stephen. Developing Critical Thinkers: Challenging Adults to Explore Alternative Ways of Thinking and Acting. San Francisco: Jossey-Bass, 1987.

Browne, Neil M.; Keeley, Stuart M. Asking the Right Questions: A Guide to Critical Thinking. New Jersey: Prentice Hall, 1986. 
Burbules, Nick C.; Berk, Rupert. "Critical Thinking and Critical Pedagogy: Relations, Differences, and Limits.» Critical Theories in Education. Hrsg. v. Thomas S. Popkewitz \& Lynn Fendler. New York: Routledge, 1999. 45-66.

Calderhead, James; Maurice Robson. «Images of Teaching: Student Teachers' Early Conceptions of Classroom Practice.» Teacher and Teacher Education, 7 (1), 1991. 1-18.

Christen, Andrea; Hofmann, Martin. Summative Produkt- und Prozessbewertung von E-Portfolios an der Pädagogischen Hochschule des Kantons St. Gallen. St. Gallen: Pädagogische Hochschule des Kantons St. Gallen, 2008: http:// api.ning.com/files/rvBadVbpvzv**YYr8x6VA6PDxTBM6yM*qOUh5sHZITIBI3 aU1Hxs9T2ZDU5bMyx19q9OWV8BhCNhfXTYAUdnB18XgzZdqaJ/Summative_Produkt_und_Prozessbewertung_von_EPortfolios_PH_St.Gallen2008.pdf (14.5.2010).

Cunningham, Ann C. «Encouraging a Reflective Disposition: Scaffolding Critical Thought Through Portfolio Development.» The Learning Portfolio: Reflective Practice for Improving Student Learning. Hrsg. v. John Zubizaretta. (2 $2^{\text {nd }}$ Edition). San Francisco: Jossey-Bass, 2009. 121-141.

Danielson, Charlotte; Abrutyn, Leslye. An Introduction to Using Portfolios in the Classroom. Alexandria Va. USA: Association for Supervision and Curriculum Development, 1997.

Dauber, Heinrich; Zwiebel, Ralf. Professionelle Selbstreflexion aus pädagogischer und psychoanalytischer Sicht. Bad Heilbrunn: Verlag Julius Klinkhardt, 2006.

Diettrich, Andreas; Meyer, Rita. "Qualifizierung und Professionalisierung des Bildungspersonals als Ansatz der Qualitätssicherung.» Qualität in Schule und Betrieb: Forschungsergebnisse und gute Praxis. Hrsg. v. Karl Wilbers, Kai Hegmann \& Thomas Bals. Aachen: Shaker-Verlag, 2008. 276-283.

Ennis, Robert H. Critical Thinking. New Jersey: Prentice Hall, 1996.

Grant, Grace E. Teaching Critical Thinking. New York: Praeger, 1988.

Greif, Siegfried. Coaching und ergebnisorientierte Selbstreflexion. Göttingen: Hogrefe, 2008.

Goodman, Jesse. «Constructing a Practical Philosophy of Teaching: A Study of Preservice Teachers' Professional Perspectives.» Teacher and Teacher Education, 4 (2), 1988. 121-137.

Gudjons, Herbert; Wagener-Gudjons, Birgit; Pieper, Marianne. Auf meinen Spuren: Übungen zur Biografiearbeit. Bad Heilbrunn: Verlag Julius Klinkhardt, 2008.

Häcker, Thomas. Portfolio: ein Entwicklungsinstrument für selbstbestimmtes Lernen. Eine explorative Studie zur Arbeit mit Portfolios in der Sekundarstufe I. 2. überarb. Aufl. Baltmannsweiler: Schneider Verlag Hohengehren, 2007.

Hewson, Peter; Beeth, Michael E.; Thorley, N. Richard. «Teaching for Conceptual Change.» International handbook of science education. Hrsg. v. Barry J. Fraser u. Kenneth Tobin. New York: Kluwer, 1998. 199-218. 
Hilzensauer, Wolf; Hornung-Prähauser, Veronika. E-Portfolio: Methode und Werkzeug für kompetenzbasiertes Lernen. Salzburg Research Forschungsgesellschaft, 2006: http://eportfolio.salzburgresearch.at/images/stories/eportfolio_ srfg.pdf (9.12.09).

Hilzensauer, Wolf. «Theoretische Zugänge und Methoden zur Reflexion des Lernens. Ein Diskussionsbeitrag.» Bildungsforschung 5.2 (2008): http://www.bildungsforschung.org/Archiv/2008-02/lernvermoegen/ (10.7.09).

Hornung-Prähauser, Veronika; Geser, Guntram; Hilzensauer, Wolf; Schaffert, Sandra. "Didaktische, organisatorische und technologische Grundlagen von E-Portfolios und Analyse internationaler Beispiele und Erfahrungen mit E-Portfolio-Implementierungen an Hochschulen.» Salzburg Research Forschungsgesellschaft (2007): http://edumedia.salzburgresearch.at/images/stories/e-portfolio_studie_srfg_fnma.pdf (22.12.2009).

Huang, Yi-Ping. "Sustaining ePortfolio: Progress, Challenges, and Dynamics in Teacher Education». Handbook of Research on ePortfolios. Hrsg. v. Ali Jafari \& Catherine Kaufman. London: Idea Group, 2006. 503-519.

Jafari, Ali; Kaufman, Catherine. Handbook of Research on ePortfolios. London: Idea Group, 2006.

Lorrainne, Stephani; Mason, Robin; Pegler, Chris. The Educational Potential of EPortfolios: Supporting Personal Development and Reflective Learning. New York: Routledge, 2007.

McDonald, James. "Curriculum, Consciousness, and Social Change.» The Hidden Curriculum and Moral Education: Deception or Discovery? Hrsg. v. Henry Giroux; David Purpel. Berkeley: McMutchan Publishing, 1983. 292-308.

McNair, Victor; Marshall, Kevin. "How ePortfolios Support Development in Early Teacher Education.» Handbook of Research on ePortfolios. Hrsg. v. Ali Jafari \& Catherine Kaufman. London: Idea Group, 2006. 474-486.

Meyers, Chet. Teaching Students to Think Critically. San Francisco: Jossey-Bass, 1986.

Moon, Jennifer. Critical Thinking: An Exploration of Theory and Practice. London: Routledge, 2008.

Nissani, Moti; Hoefler-Nissani, Donna Marie. «Experimental Studies of Belief Dependence of Observations and of Resistance to Conceptual Change.» Cognition \& Instruction, 9 (2), 1992. 97-111.

O.A. (2009a). «Current SCIL Research Projects.» Stanford Centre for Innovations in Learning: http://scil.stanford.edu/research/projects_by_title.html (22.12.09).

O.A. (2009b). «E-Portfolios.»E-Learning-Portal Baden Württemberg: http://www.elearning-bw.de/unterricht-gestalten/e-szenarien/e-portfolios.html (23.12.09).

Offermanns, Martina. Braucht Coaching einen Coach? - Eine evaluative Pilotstudie. Stuttgart: ibidem-Verlag, 2004. 
Pajares, Frank M. «Teachers' Beliefs and Educational Research: Cleaning Up a Messy Construct.» Review of Educational Research, 62 (3), 1992. 307-332.

Paul, Richard. Critical Thinking. What every person needs to survive in a rapidly changing world. (3. Ausg.). Dillon Beach: Foundation for Critical Thinking, 1993.

Peters, Martine; Chevrier, Jaques; LeBlanc, Raymond; Fortin, Gilles; Malette, Judith. «The ePortfolio: A Learning Tool for Pre-Service-Teachers». Handbook of Research on ePortfolios. Hrsg. v. Ali Jafari \& Catherine Kaufman. London: Idea Group, 2006. 313-326.

Petri, Gottfried. Kritisches Denken als Bildungsaufgabe und Instrument der Schulentwicklung. Innsbruck: Studienverlag, 2003.

Preckel, Daniel; Aschwanden, Nora Lichti. «Weiterbildung von Richterinnen und Richtern im Kanton Zürich mit Hilfe eines virtuell unterstützten Lernportfolios.» Handbuch E-Learning (31. Ergänzungslieferung, 8.38. Hrsg. v. A. Hohenstein \& K. Wilbers. Köln: Deutscher Wirtschaftsdienst, 2010. 1-14.

Richter, Jonathon, J. «Future-Focused ePortfolios at Montana State University». Handbook of Research on ePortfolios. Hrsg. v. Ali Jafari \& Catherine Kaufman. London: Idea Group, 2006. 551-557.

Schratz, Michael. «Teacher Professionality: Kooperation zwischen Universitäten und Pädagogischen Hochschulen im europäischen Kontext.» Universität Wien, 2006: http://spl.univie.ac.at/fileadmin/user_upload/bologna/Tagung_ Lehramt/061107-Tagung-LA-Schratz.pdf (10.7.09).

Schaub, Harald. «Störungen und Fehler beim Denken und Problemlösen.» Universität Heidelberg (o. J.): http://www.psychologie.uni-heidelberg.de/ae/allg/ enzykl_denken/Enz_09_Schaub.pdf (10.7.09).

Spronken-Smith, Rachel; Stein, Sarah. "Challenging Tertiary Teachers' Beliefs and Practices: Facilitating Change and Development Through Portfolios.» The Learning Portfolio: Reflective Practice for Improving Student Learning. Hrsg. v. John Zubizaretta. $2^{\text {nd }}$ Edition. San Francisco: Jossey-Bass, 2009. 197-211.

Tisdale, Tim. Selbstreflexion, Bewussstein und Handlungsregulation. Weinheim: Beltz-Verlag, 1998.

Trager, Bernhard. Selbstreflexionsprozsse im Coaching: Die Auswirkungen auf Klienten und deren Umfeld als Einflussfaktoren auf den Coaching-Erfolg. Saarbrücken: VDM-Verlag, 2008.

Trenkamp, Oliver. «Ungerechte Grundschullehrer. Auch der intelligente Kevin ist dumm dran.» Spiegel Online 18.9.2009: http://www.spiegel.de/schulspiegel/ wissen/0,1518,649736,00.html (19.11.09).

Tusch Hanspeter; Steinlechner, Manfred. Selbstreflexionstraining in der Lehrerbildung. Innsbruck: Golf-Verlag, 1988.

Vosniadou, Stella (Hrsg.). International Handbook of Research on Conceptual Change. New York: Routledge, 2008. 
Weil, Danny. "Assumptions. Believing and Knowing.» Critical Thinking and Learning: An Encyclopedia for Parents and Teachers. Hrsg. v. Joe. L. Kincheloe; Danny Weil. London: Greenwood Press, 2004. 63-67.

Weinz, Rafaela (2003). «Wie Schule erzieht: Der geheime Lehrplan.» Weinzweb: http://www.weinzweb.de/rw/Der_Geheime_Lehrplan.pdf (14.1.09)

Wideen, Marvin; Mayer-Smith, Jolie; Moon, Barbara. "A Critical Analysis of the Research on Learning to Teach: Making the Case for an Ecological Perspective on Inquiry.» Review of Educational Research, 68 (2), 1998. 130-178.

Wright, Alan; Hartley, M. Heather. «Upon Further Review: A Second Look at the Student Learning Portfolio.» The Learning Portfolio: Reflective Practice for Improving Student Learning. Hrsg. v. John Zubizaretta. $2^{\text {nd }}$ Edition. San Francisco: Jossey-Bass, 2009. 223-238.

Yang, Ya-Ting. "A Catalyst for Teaching Critical Thinking in a Large University Class in Taiwan: Asynchrous Online Discussions with the Facilitation of Teaching Assistants.» Educational Technology Research and Development, III (56), Juni 2007. 241-264. 